Supplemental Information

\title{
Spatiotemporal characterization of GPCR activity and function during endosomal trafficking pathway
}

Hyunbin $\mathrm{Kim}^{1,2}$, Hae Nim Lee ${ }^{1,3}$, Jaesik Choi $^{4 *}$ and Jihye Seong ${ }^{1-3 *}$

${ }^{1}$ Brain Science Institute, Korea Institute of Science and Technology (KIST), Seoul, 02792, Republic of Korea

${ }^{2}$ Division of Bio-Medical Science \& Technology, KIST School, Korea University of Science and Technology, Seoul, 02792, Republic of Korea

${ }^{3}$ Department of Converging Science and Technology, Kyung Hee University, Seoul, 02447, Republic of Korea

${ }^{4}$ Graduate School of Artificial Intelligence, Korea Advanced Institute of Science and Technology (KAIST), Daejeon, 34141, Republic of Korea

*Correspondence: jseong@kist.re.kr; jaesik.choi@kaist.re.kr 


\section{Table of contents}

Figure S1. Improved distribution of the FRET-based B2AR biosensor ………….................S3

Figure S2. The B2AR activity at the internalized endosomes, monitored by FRET-based B2AR biosensor

Figure S3. Time-lapse changes of the FRET/CFP ratios in the HEK cells expressing B2AR-Gs or

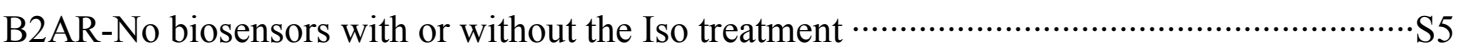

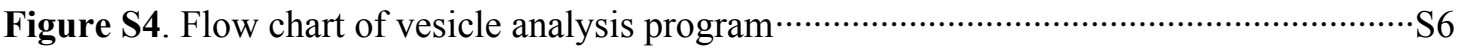

Figure S5. Co-localization test of the agonist-stimulated B2AR biosensor with endosomal markers in live cells S7

Figure S6. Amino acid sequences of B2AR biosensor, B2AR-pH sensor, pF-Rab5a, pF-Rab7a and LAMP2-pF S8

\section{Legends for Supplementary movies:}

Movie S1. Time-lapse imaging of B2AR FRET biosensor in response to the Iso treatment $\cdot$ S9

Movie S2. Automatic vesicle detection by Vesicle-Tracker S9 
a)
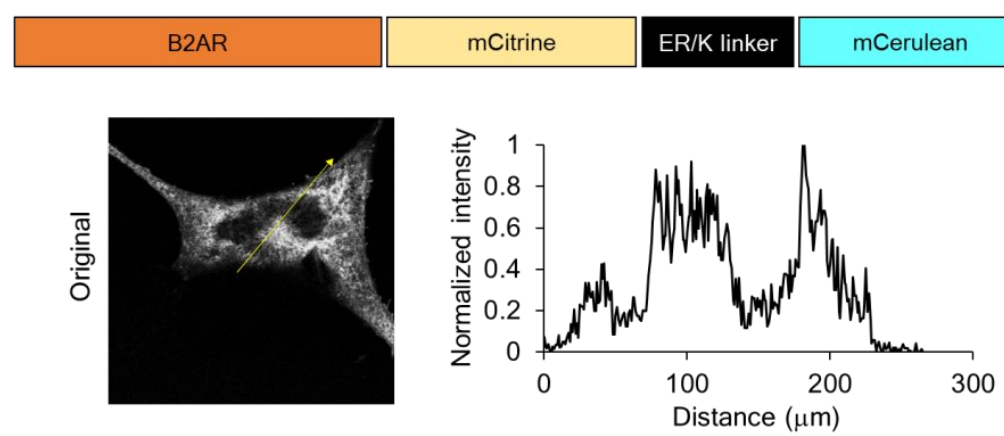

b)

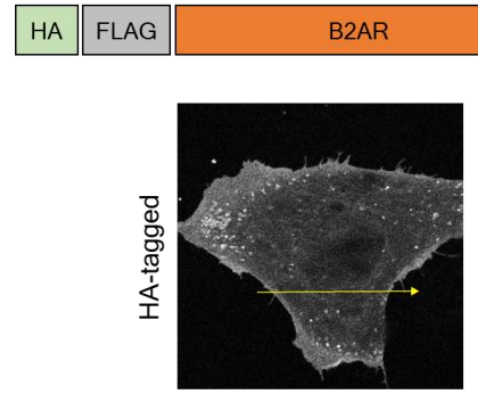

mCitrine

ER/K linker

mCerulean

Gs peptide

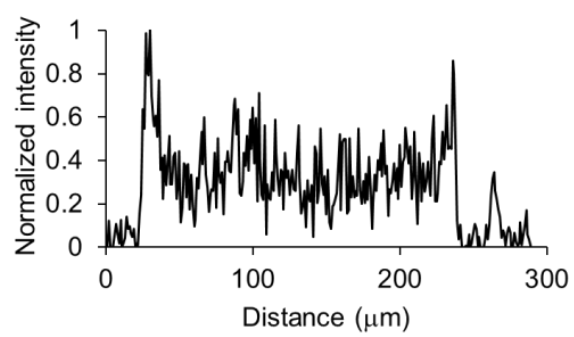

\section{Figure S1. Improved distribution of the FRET-based B2AR biosensor}

(a) The top panel shows the scheme of original B2AR FRET. The bottom panels show the representative mCitrine confocal images of the original B2AR FRET biosensor (left), and the normalized intensity along the yellow arrowed lines of the left image (right). (b) The top panel shows the scheme of the B2AR FRET with an insertion of signal peptide of HA and a FLAG epitope in its $\mathrm{N}$-terminus. The bottom panels show the representative mCitrine confocal images of this B2AR FRET biosensor (left), and the normalized intensity along the yellow arrowed lines of the left image (right). 
a)

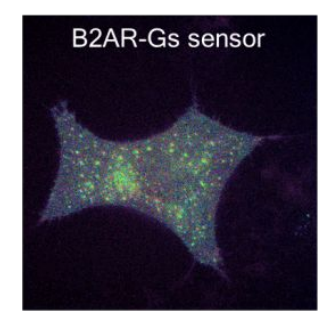

b)

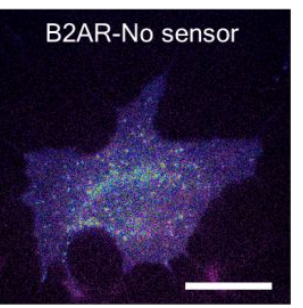

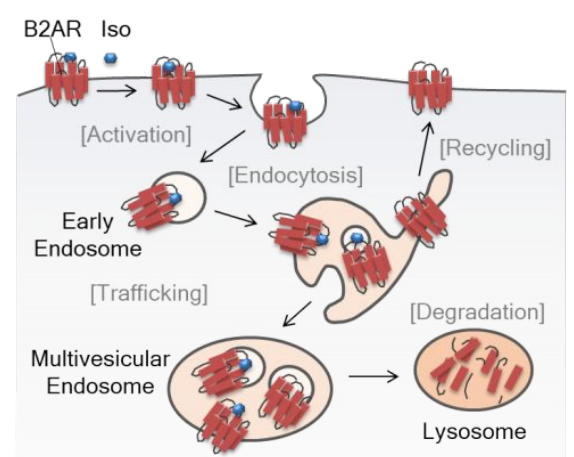

Figure S2. The B2AR activity at the internalized endosomes, monitored by FRET-based

\section{B2AR biosensor}

(a) Representative images of B2AR-Gs biosensor and B2AR-No biosensor (negative control biosensor missing Gs peptide in the C-terminus) after 1 hour of the $10 \mu \mathrm{M}$ Iso treatment. The rainbow color bar on the right represents the level of FRET/CFP emission ratio. Both biosensors are internalized, but only B2AR-Gs biosensor reports the high FRET/CFP ratio. Scale bar, $20 \mu \mathrm{m}$. (b) Scheme of endosomal trafficking pathway of the agonist-stimulated GPCR, for example Iso-induced B2AR activation. 


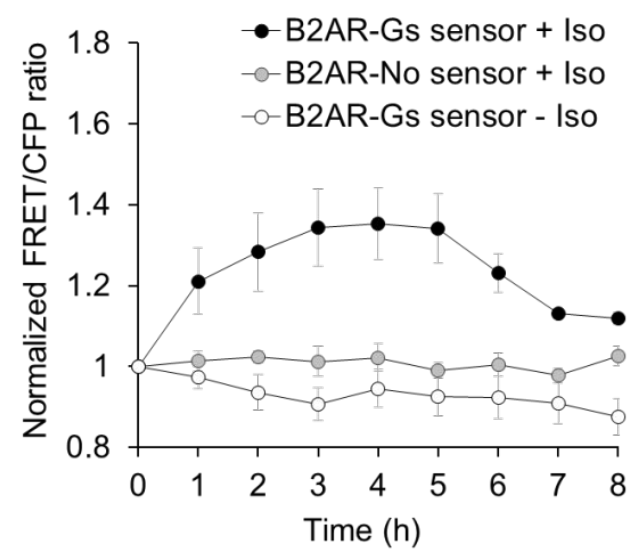

Figure S3. Time-lapse changes of the FRET/CFP ratios in the HEK cells expressing B2AR-Gs or B2AR-No biosensors with or without the Iso treatment.

The FRET/CFP ratios of whole cell regions were analyzed, and data represent the mean value \pm s.e.m. of each group experiment ( $\mathrm{n}=8$ or 9$)$. 

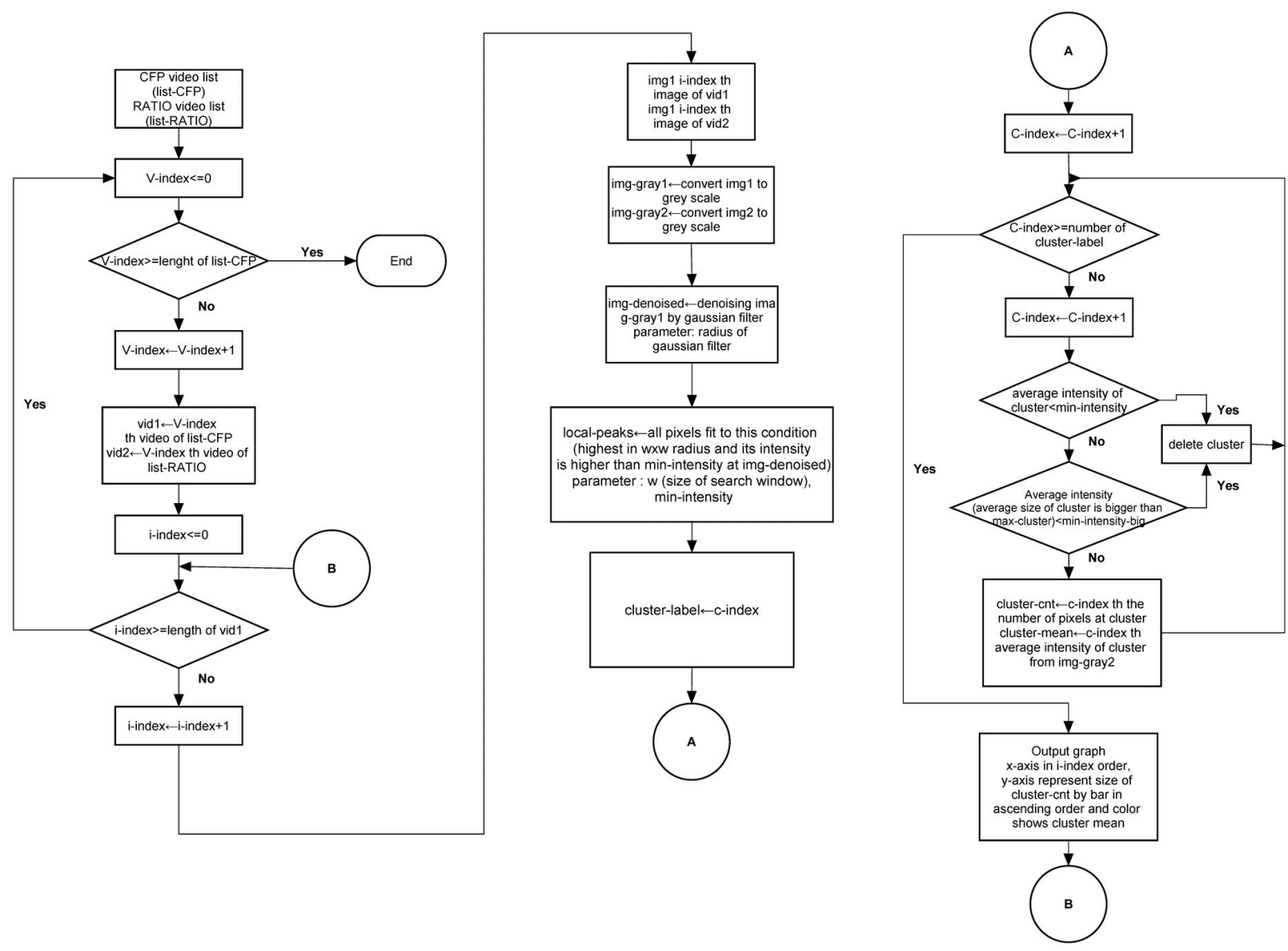

Figure S4. Flow chart of vesicle analysis program

Flow chart shows algorithm applied to identify and analyze the FRET/CFP ratio of B2AR biosensor at the level of individual endosomes. 
a)
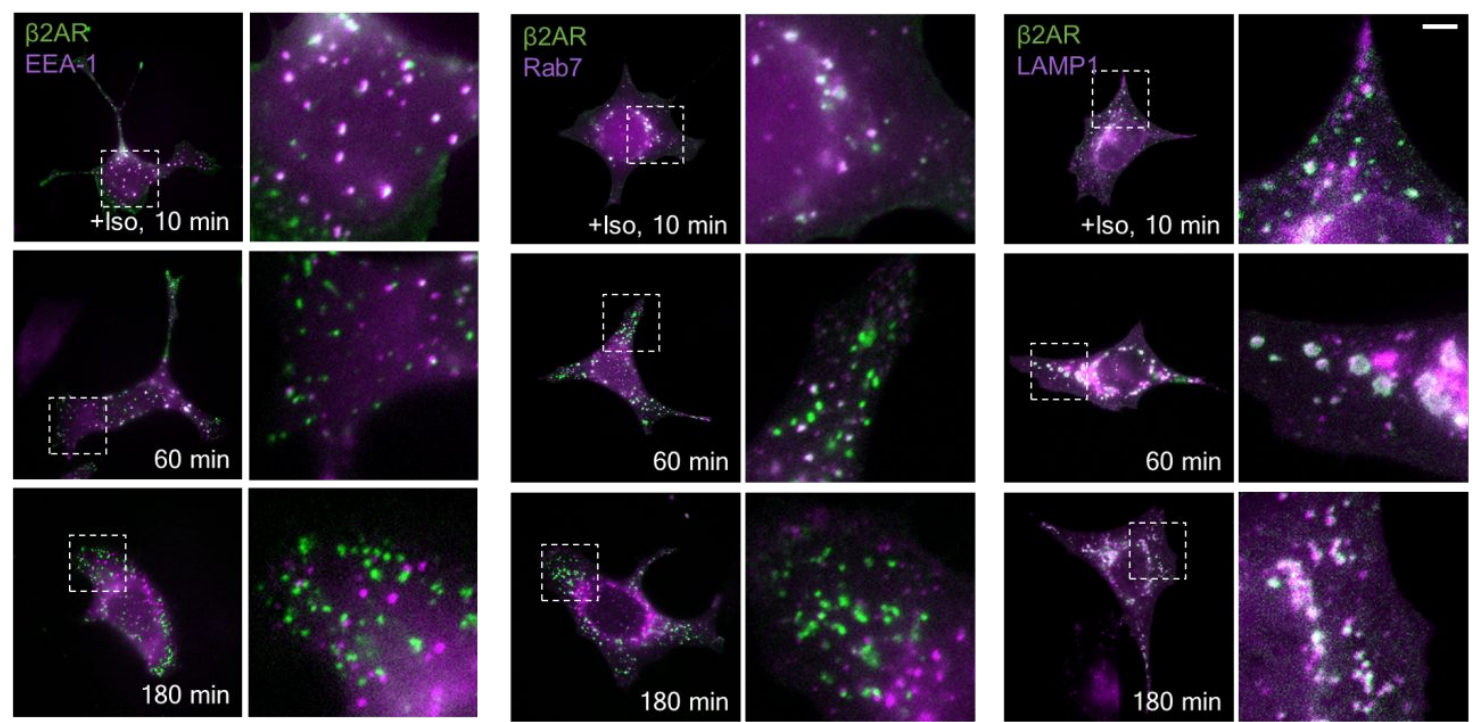

b)

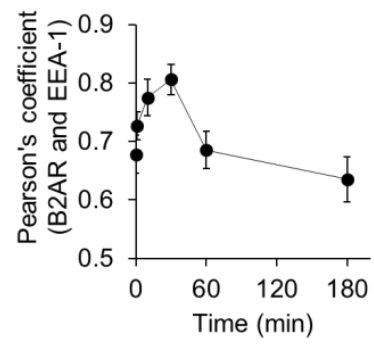

c)

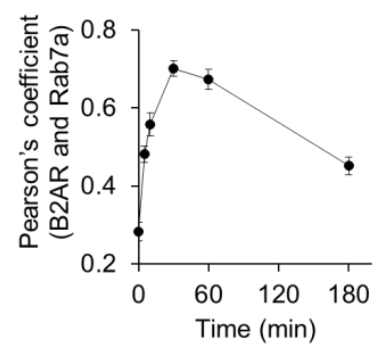

d)

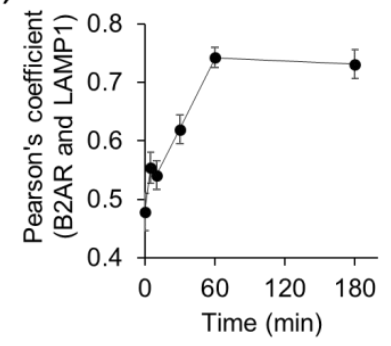

Figure S5. Co-localization test of the agonist-stimulated B2AR biosensor with endosomal markers in live cells

(a) Representative images of B2AR biosensor (green) and trafficking markers (magenta) at indicated times after the Iso stimulation. Pearson's coefficient between B2AR and (b) early endosome marker (EEA-1) (n=17-26), (c) late endosome/MVB marker (Rab7a) (n=21-30) or (d) lysosome marker (LAMP-1) $(n=10-25)$. Data represent the mean value \pm s.e.m. of experiments. 
B2AR biosensor:

MKTIIALSYIFCLVFADYKDDDDASIDMGQPGNGSAFLLAPNRSHAPDHDVTQQRDEVWVVGMGIVMSLIVLAIVFGNVLVITAIAKFERLQ TVTNYFITSLACADLVMGLAVVPFGAAHILMKMWTFGNFWCEFWTSIDVLCVTASIETLCVIAVDRYFAITSPFKYOSLLTKNKARVIILMV WIVSGLTSFLPIQMHWYRATHQEAINCYANETCCDFFTNQAYAIASSIVSFYVPLVIMVFVYSRVFQEAKRQLQKIDKSEGRFHVQNLSQVE QDGRTGHGLRRSSKFCLKEHKALKTLGIIMGTFTLCWLPFFIVNIVHVIQDNLIRKEVYILLNWIGYVNSGFNPLIYCRSPDFRIAFQELLCLRR SSLKAYGNGYSSNGNTGEQSGYHVEQEKENKLLCEDLPGTEDFVGHQGTVPSDNIDSQ GRNCSTNDSLLGSGSGGSGGSGGSGVSKGEELF TGVVPILVELDGDVNGHKFSVSGEGEGDATYGKLTLKFICTTGKLPVPWPTLVTTFGYGLMCFARYPDHMKQHDFFKSAMPEGYVQERTIH FKDDGNYKTRAEVKFEGDTLVNRIELKGIDFKEDGNILGHKLEYNYNSHNVYIMADKQKNGIKVNFKIRHNIEDGSVQLADHYQQNTPIGD GPVLLPDNHYLSYQSKLSKDPNEKRDHMVLLEFVTAA GITLGMDELYKEFGSGGSGENLYFQGGSGEEEEKKKQQEEEAERLRRIQEEMEK ERKRREEDEQRRRKEEEERRMKLEMEAKRKQEEEERKKREDDEKRKKKGSGGSGGAPVSKGEELFTGVVPILVELDGDVNGHKFSVSGEG EGDATYGKLTLKFICTTGKLPVPWPTLVTTLTWGVQCFARYPDHMKQHDFFKSAMPEGYVQERTIFFKDDGNYKTRAEVKFEGDTLVNRI ELKGIDFKEDGNILGHKLEYNAISDNVYITADKQKNGIKANFKIRHNIEDGSVQLADHYQQNTPIGDGPVLLPDNHYLSTQSKLSKDPNEKR DHMVLLEFVTAAGITLGMDELYKLINGSGGSGGSGDTENIRRVFNDCRDIIQRMHLRQYELL*

Green: HA signal peptide, Grey: FLAG, Orange: B2AR, Yellow: mCitrine, Navy: ER/K linker, Cyan: mCerulean, Red: C-terminal peptide of Gs protein

\section{B2AR-pH sensor:}

MKTIIALSYIFCLVFADYKDDDDASIDMGQPGNGSAFLLAPNRSHAPDHDVTQQRDEVWVVGMGIVMSLIVLAIVFGNVLVITAIAKFERLQ TVTNYFITSLACADLVMGLAVVPFGAAHILMKMWTFGNFWCEFWTSIDVLCVTASIETLCVIAVDRYFAITSPFKYQSLLTKNKARVIILMV WIVSGLTSFLPIQMHWYRATHQEAINCYANETCCDFFTNQAYAIASSIVSFYVPLVIMVFVYSRVFOEAKROLQKIDKSEGRFHVQNLSQVE QDGRTGHGLRRSSKFCLKEHKALKTLGIIMGTFTLCWLPFFIVNIVHVIQDNLIRKEVYILLNWIGYVNSGFNPLIYCRSPDFRIAFQELLCLRR SSLKAYGNGYSSNGNTGEQSGYHVEQEKENKLLCEDLPGTEDFVGHQGTVPSDNIDSQGRNCSTNDSLLGSGSGGSGGSGGSGVSKGEENN MAIIKEFMRFKVRMEGSVNGHEFEIEGEGEGRPYEGTQTAKLKVTKGGPLPFAWDILSPQFTYGSKAYVKHPADIPDYLKLSFPEGFKWERV MNFEDGGVVTVTQDSSLQDGEFIYKVKLRGTNFPSDGPVMQKKTMGWEASTERMYPEDGALKGEIKMRLKLKDGGHYDAEVKTTYMAK KPVQLPGAYKTDIKLDITSHNEDYTIVEQYERAEGRHSTGGMDELYKGGGGSMVSKGEELFTGVVPILVELDGDVNGHKFSVSGEGEGDA YGKLTLKFICTTGKLPVPWPTLVTTLTYGVOCFSRYPDHMKOHDFFKSAMPEGYVOERTIFFKDDGNYKTRAEVKFEGDTLVNRIELKGIDF KEDGNILGHKLEYNYNSHNVYIMADKQKNGIKVNFKIRHNIEDGSVQLADHYQQNTPIGDGPVLLPDNHYLSTQSALSKDPNEKRDHMVL LEFVTAAGITLGMDELYK*

Green: HA signal peptide, Grey: FLAG, Orange: B2AR, Red: pHTomato, Neon green: EGFP

pF-Rab5a:

MLVSKGEEDNMAIIKEFMRFKVHMEGSVNGHEFEIEGEGEGRPYEAFQTAKLKVTKGGPLPFAWDIL SPQFMYGSKAYIKHPADIPDYFKLS FPEGFRWERVMNFEDGGIIHVNQDSSLQDGVFIYKVKLRGTNFPPDGPVMQKKTMGWEQWRDPPLLSQRGPDALLTVALRKPPGQRTDEE LDLIFEELLHIKAVAHLSNSVKRELAAVLLFEPHSKAGTVLFSQGDKGTSWYIIWKGSVNVVTHGKGLVTTLHEGDDFGQLALVNDAPRAA TILRENNCHFLRVDKODFNRIIKDVEFKVETVDERMYPEDGALKSEIKKGLRLKDGGHYAAEVKTTYKAKKPVQLPGAYIVDIKLDIVSHN EDYTIVEQCERAEGRHSTGGMDELYKSGLRSRAQMASRGATRPNGPNTGNKICQFKLVLLGESAVGKSSLVLRFVKGQFHEFQESTIGAAF LTQTVCLDDTTVKFEIWDTAGQERYHSLAPMYYRGAQAAIVVYDITNEESFARAKNWVKELQRQASPNIVIALSGNKADLANKRAVDFQE AQSYADDNSLLFMETSAKTSMNVNEIFMAIAKKLPKNEPQNPGANSARGRGVDLTEPTQPTRNQCCSNTRTRPLEQKLISEEDL*

Magenta: pink Flamindo, Brown: Rab5a, Blue: myc tag

pF-Rab7a:

MLVSKGEEDNMAIIKEFMRFKVHMEGSVNGHEFEIEGEGEGRPYEAFQTAKLKVTKGGPLPFAWDILSPQFMYGSKAYIKHPADIPDYFKLS FPEGFRWERVMNFEDGGIIHVNQDSSLQDGVFIYKVKLRGTNFPPDGPVMQKKTMGWEQWRDPPLLSQRGPDALLTVALRKPPGQRTDEE LDLIFEELLHIKAVAHLSNSVKRELAAVLLFEPHSKAGTVLFSOGDKGTSWYIIWKGSVNVVTHGKGLVTTLHEGDDFGQLALVNDAPRAA TIILRENNCHFLRVDKQDFNRIIKDVEFKVETVDERMYPEDGALKSEIKKGLRLKDGGHYAAEVKTTYKAKKPVQLPGAYIVDIKLDIVSHN EDYTIVEQCERAEGRHSTGGMDELYKSGLRSRAQMTSRKKVLLKVIILGDSGVGKTSLMNQYVNKKFSNQYKATIGADFLTKEVMVDDRL VTMQIWDTAGQERFOSLGVAFYRGADCCVLVFDVTAPNTFKTLDSWRDEFLIQASPRDPENFPFVVLGNKIDLENRQVATKRAQAWCYSK NNIPYFETSAKEAINVEQAFQTIARNALKQETEVELYNEFPEPIKLDKNDRAKASAESCSCTRTRPLEQKLISEEDL*

Magenta: pink Flamindo, Brown: Rab7a, Blue: myc tag

\section{LAMP2-pF:}

MVCFRLFPVPGSGLVLVCLVLGAVRSYALELNLTDSENATCLYAKWOMNFTVRYETTNKTYKTVTISDHGTVTYNGSICGDDQNGPKIAV QFGPGFSWIANFTKAASTYSIDSVSFSYNTGDNTTFPDAEDKGILTVDELLAIRIPLNDLFRCNSLSTLEKNDVVQHYWDVLVQAFVQNGTV STNEFLCDKDKTSTVAPTIHTTVPSPTTTPTPKEKPEAGTYSVNNGNDTCLLATMGLQLNITQDKVASVININPNTTHSTGSCRSHTALLRLNS STIKYLDFVFAVKNENRFYLKEVNISMYLVNGSVFSIANNNLSYWDAPLGSSYMCNKEQTVSVSGAFQINTFDLRVQPFNVTQGKYSTAQE CSLDDDTILIPIIVGAGLSGLIIVIVIAYVIGRRKSYAGYOTLGSTGSTGSTGAVDGTAGPGSIATMLVSKGEEDNMAIIKEFMRFKVHMEGSV NGHEFEIEGEGEGRPYEAFQTAKLKVTKGGPLPFAWDILSPQFMYGSKAYIKHPADIPDYFKLSFPEGFRWERVMNFEDGGIIHVNQDSSLQ DGVFIYKVKLRGTNFPPDGPVMQKKTMGWEQWRDPPLLSQRGPDALLTVALRKPPGQRTDEELDLIFEELLHIKAVAHLSNSVKRELAAVL LFEPHSKAGTVLFSQGDKGTSWYIIWKGSVNVVTHGKGLVTTLHEGDDFGQLALVNDAPRAATIILRENNCHFLRVDKQDFNRIIKDVEFK VETVDERMYPEDGALKSEIKKGLRLKDGGHYAAEVKTTYKAKKPVQLPGAYIVDIKLDIVSHNEDYTIVEQCERAEGRHSTGGMDELYK*

Brown: LAMP2B, Magenta: pink Flamindo

Figure S6. Amino acid sequences of B2AR biosensor, B2AR-pH sensor, pF-Rab5a, pF-

\section{Rab7a and LAMP2-pF}


Video S1. Time-lapse imaging of B2AR FRET biosensor in response to the Iso treatment.

Video S2. Automatic endosome detection by vesicle detection program. The endosomal vesicles were detected from a CFP image (left) of the B2AR biosensor (right). The detected vesicles were displayed by pink dots. 